From the observed doublet separation in terms of one-electron moleoules $(\mathrm{ZnH}, \mathrm{CdH}, \mathrm{HgH})$ the strength of the magnetic field, induced by the rotation in BiH, may be estimated, and it was found that a com. plete Paschen-Back effect appeared already for $K>3$, causing the disappearance of the hyperfine structure. This behaviour of the model corresponds to the observations noted above. For large $K$-values (case $b^{\prime}$, also including ${ }^{1 \Sigma}$-terms) a kind of rotational hyperfine structure should appear, due to an orientation of the nuclear spin to the axis of rotation. In $1 \Sigma^{*} \rightarrow 1 \Sigma$ the calculated width of lines due to this effect, $\sigma i_{k} g_{i}$ $\left(i_{K}=+i, i-1, \ldots-i\right)$, amounts to $\sim 0.02 \mathrm{~A}$., which, however, is still too small to be distinguished from other factors governing the line width. It would appear from what is said above, that the $\mathrm{BiH}$ molecule is not a favourable one for such observations ; lighter molecules are to be preferred. I also wish to emphasise that my interpretation of the effect is not observed, the hyperfine structure of the band lines not being resolved.

In addition, we must take into consideration that in some atoms deviating properties in the electric field of their isotopes have been observed, as clearly indicated in the case of neon. It is difficult to overlook the molecular effect resulting from this factor. Investigations are being pursued on both sides of this problem.

Laboratory of Physies,

University of Stockholm, Dec. 4.

2 S. Mrozowski, Zeit. für Phys., 72, 776; 1931

2 B. A. Brice, Phys. Rev., 35, $966 ; 1930$.

* R. Rydberg, Zeit. für Phys., 73, 74; 1931

- A. Heimer and $\mathrm{E}$. Hulthen, NATURE, 127, 537; 1931.

\section{Significance of Velocity Measurements in Relation to the Benzene Substitution Problem.}

From the results obtained by Holleman, ${ }^{1}$ Hückel ${ }^{2}$ has calculated the relative action constants and the differences between the energies of activation for the nitration of certain benzene derivatives in the ortho, meta, and para positions. The energy of activation for the ortho position is found to be greater than that for the para, and at the temperature used the amount of ortho derivative formed is smaller than that of para in the case of chlor-and brom-benzene, and the exception found in the case of toluene may be met by allowing for the double opportunity of ortho sub. stitution as compared with that of para. These results are interesting from the point of view of steric hindrance. The equality of the action constants taken in conjunction with the facts given above shows that the lower rate of reaction observed for the ortho position is not due to steric hindrance acting on an activated molecule but to an actual paucity of activated molecules due to a difference in activation energy.

The close connexion between substitution problems and the reactivity of substituents attached to the benzene nucleus encourages me to report here the results of some experiments on the velocity of reaction between ortho, meta, and para toluidines and benzyl chloride. I have found that the energy of activation of the ortho compound is distinctly higher than that for the para compound, so that here, as in the case of direct substitution, the lower rate of reaction is not due to steric hindrance acting on activated molecules but to a paucity of active molecules. The steric effect, then, of the methyl group in the above case acts directly on the nitrogen atom by increasing the value of the energy necessary to bring it to the activated state. There is thus apparently a sort of co-ordination between the methyl group and the amino group, of a kind possibly similar to that postulated by Sidgwick in similar cases.

Where three atoms or groups, $A, B$, and $C$, are present in the vicinal or $1: 2: 3$ positions, then if $B$ is exerting an effect of this kind on $C$ the presence of $A$-if its demand is greater than that of hydrogenby sharing and so reducing the effect of $B$ may raise the reactivity of $C$; this kind of effect was observed by Menschutkin in the case of vicinal $o$-xylidine. Other evidence of something in the nature of direct chemical interaction between the methyl and amino groups in ortho toluidine and its derivatives is the way in which ortho toluidine frequently imitates the behaviour of secondary bases and monoalkyl derivatives imitate tertiary bases. To return to the general case, when $A$ or $C$ is a hydrogen atom, the case reduces to the substitution of a disubstituted benzene derivative.

A vicinal effect of a different kind was observed by Holleman ${ }^{3}$ in the case of 1 nitro 2: 6 dichlorbenzene, which may possibly be explained by a sharing of the activating effect of the nitro group.

There is, however, another point worthy of consideration. If the action constant were really a function of the form $a e^{-b / T}$ then the effect of a variation in $b$ would only be shown in the so-called energy of activation, the variation in which might be only partially due to a change in the real energy of activation. In the case of ortho toluidine, for example, the life of the activated amino group-which would presumably affect the action constant-might be appreciably affected by the ease of interchange of activation energy between the amino group and the adjacent methyl group, and if the probability of this interchange were of the required form then the observed energy of activation would contain a disguised "action constant 'term. It would appear that the question of the factors governing the lives of active molecules is one of primary interest in this connexion; a kind of molecular insurance office is, in fact, required.

I may add in conelusion that the activation energy of meta toluidine was of the order predicted by the Lapworth-Robinson hypothesis, and that these experiments will be resumed shortly.

University College, Rangoon,

D. H. Peacock. Burma.

1 Rev. Tran, Chim., 18, 267, et seq. ; 1899.

"Theoretische Grundiagen der organischen Chemie", vol. 2, p. 255 ${ }^{3}$ Rev. Tran. Chim., 35, 1; 1915 .

\section{Hall Effect and Superconductivity.}

Tur Hall effect of the so-called electronic semiconductors is usually found to be comparatively very large. All the semiconductors investigated up to the present time obey this rule, with the exception of load sulphide (PbS), for which the experimental data vary from one sample to another. For example, according to van Aubel ${ }^{1}$ and Heaps, ${ }^{2}$ chemically pure lead sulphide has a very small Hall coefficient, while the Hall constant of the natural lead sulphide (galena) may (according to investigations by $\mathrm{A}$. Smith ${ }^{3}$ and Heaps ${ }^{2}$ ) reach values as large as 254 .

We have studied the Hall effect of copper sulphide (CuS) prepared by exposing a copper plate to sulphur vapour. ${ }^{4,5}$. The Hall coefficient was found to be less than $10^{-3}$ c.g.s. units.

It is well known that copper and lead sulphides are semiconductors which belong to the class of superconductors. It was natural to consider the question whether the small values of the Hall coefficient may be related to the existence of superconductivity.

The experimental data on the Hall effect in superconductors seem to show that this correlation really

No. 3245 , VoL. 129] 